\title{
FINITE ELEMENT MODELLING OF 3-3 PIEZOCOMPOSITES
}

\author{
A. Perry and C.R. Bowen \\ Department of Materials Science \& Engineering, University of Bath, Bath, Somerset, UK, \\ BA2 7AY \\ S.W. Mahon \\ Mechanical Sciences Sector, DERA Farnborough, Hampshire, UK, GU14 OLX
}

(Received June 18, 1999)

(Accepted June 28, 1999)

Keywords: Computer simulation; Ceramics; Piezoelectricity; Acoustic; Piezocomposite

\section{Introduction}

Efforts to improve the properties and reliability of piezoelectric materials have often concentrated on processing dense ceramic material by techniques such as fine particle size powder production, grain size control, hot pressing, hot isostatic pressing and controlled atmosphere sintering [1-3]. However, it has been shown that lowering the density and introducing significant amounts of open porosity into a piezoelectric material can lead to large improvements in its performance for specific applications, such as low-frequency hydrophones [4-8]. Due to the microstructural connectivity, these open porosity structures are termed 3-3 piezoelectric composites or piezocomposites. In this convention the first digit refers to the connectivity of the active piezoelectric phase while the second digit refers to the inactive phase, which is air or a flexible polymer [9].

The interest in piezocomposite materials is due to a demand for low frequency hydrophone/actuator devices for use in underwater acoustic systems typical of those encountered in Navy sonar array applications and also in civilian applications such as oil reservoir monitoring, seismic monitoring, bio-acoustic research and general oceanographic applications [10]. Parameters to consider for hydrophone applications are sensitivity, impedance, depth capability, frequency range and cost.

\section{$\underline{\text { Sensitivity }}$}

At low frequencies $(<100 \mathrm{kHz})$ the wavelength dimensions are much greater than those of the hydrophone and the stress on the device due to the acoustic wave is effectively hydrostatic [11]. Under these conditions, passive hydrophone sensitivity is generally characterised by the hydrostatic piezoelectric voltage constant $\left(\mathrm{g}_{\mathrm{h}}\right)$, defined as the electric field generated per unit hydrostatic pressure [12], where

$$
\begin{aligned}
& \mathrm{g}_{\mathrm{h}}=\mathrm{d}_{\mathrm{h}} / \epsilon^{\mathrm{T}}{ }_{33}\left(\mathrm{~V} \mathrm{mPa}^{-1}\right) \\
& \epsilon_{33}^{\mathrm{T}}=\text { permittivity at constant stress }\left(\mathrm{F} \mathrm{m}^{-1}\right) \\
& \mathrm{d}_{\mathrm{h}}=\text { hydrostatic strain constant }\left(\mathrm{m} \mathrm{V}^{-1}\right)
\end{aligned}
$$




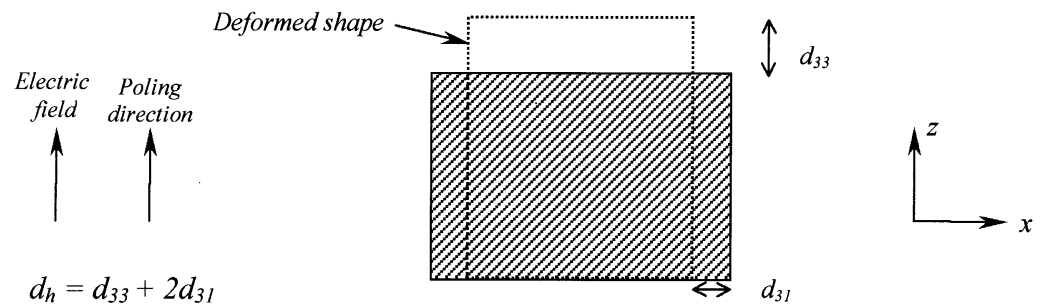

Figure 1. Schematic diagram of an electric field applied to a dense piezoelectric material. The high lateral coupling results in a low $d_{h}$ and the additional high permittivity of the ceramic results in a low $g_{h}$.

The hydrostatic strain constant $\left(\mathrm{d}_{\mathrm{h}}\right)$ indicates the performance of a material as an actuator in the active mode [13] and is defined as the hydrostatic strain per unit electric field, where

$\mathrm{d}_{\mathrm{h}}=\mathrm{d}_{33}+\mathrm{d}_{31}+\mathrm{d}_{32}$

$=\mathrm{d}_{33}+2 \mathrm{~d}_{31}\left(\right.$ since $\left.\mathrm{d}_{31}=\mathrm{d}_{32}\right)$

$\mathrm{d}_{33}=$ strain per unit field (or charge per unit force) parallel to the direction of the applied electric field $(\mathrm{z}),\left(\mathrm{m} \mathrm{V}^{-1}\right)$

$\mathrm{d}_{31}, \mathrm{~d}_{32}=$ strain per unit electric field normal to the direction of the electric field ( $\left.\mathrm{x}, \mathrm{y}\right),\left(\mathrm{m} \mathrm{V}^{-1}\right)$.

For bulk, dense materials $d_{h}$ is often low, due to the fact that $d_{33}$ and $d_{31}$ are both large but opposite in sign. In physical terms, as the piezoelectric expands in the poling direction $(\mathrm{z})$ by an electric field (due to the positive value of $\mathrm{d}_{33}$ ) there is also a contraction in its $\mathrm{x}$ and $\mathrm{y}$ dimensions (due to the negative sign of $\mathrm{d}_{31}$ ) as shown schematically in Figure 1 . The aim of a piezocomposite is to create a structure in which the effective $d_{31}$ is reduced while retaining the $d_{33}$, i.e. the actuation becomes increasingly 'piston-like' and of high $\mathrm{d}_{\mathrm{h}}$. This is the principal mechanism in a 1-3 piezocomposite whereby thin rods of piezoelectric material are embedded in a polymer matrix [13-15]. In addition to the low $\mathrm{d}_{\mathrm{h}}$, the figure of merit $\mathrm{g}_{\mathrm{h}}\left(=\mathrm{d}_{\mathrm{h}} / \epsilon^{\mathrm{T}}{ }_{33}\right)$ of dense piezoceramics is further reduced by the high permittivity of these materials.

The 3-3 piezocomposites have both interconnected porosity and ceramic phases and are fabricated most cost effectively by producing highly porous ceramic structures. As porosity is introduced into a 3-3 microstructure there is a reduction in permittivity $\left(\epsilon_{33}\right)$ as low permittivity polymer or air replaces the high permittivity ceramic. There is also an increase in the hydrostatic strain constant $\left(d_{h}\right)$ [11]. These two changes result in a material with a much higher $\mathrm{g}_{\mathrm{h}}$ value, up to $100 \times 10^{-3} \mathrm{~V} \mathrm{mPa}^{-1}$ compared to $2 \times 10^{-3} \mathrm{~V} \mathrm{mPa}^{-1}$ for a bulk material [11]. The hydrostatic figure of merit $\mathrm{d}_{\mathrm{h}} \cdot \mathrm{g}_{\mathrm{h}}$, the parameter which describes the performance of the device as a hydrophone and an actuator (an active-passive transducer) also increases with increasing porosity. This can be an order of magnitude greater than for 1-3 piezocomposites.

\section{Acoustic Impedance}

The decrease in density of 3-3 composites compared to the bulk material lowers the elastic modulus and acoustic impedance which results in better impedance matching of the composite with water and biological tissue. For example, 7MRayl has been measured for some porous materials compared to 30MRayl for the bulk material [16]. This removes the need for impedance matching layers, which are required when dense piezoceramics are used. A lower density also reduces the total weight of the device, which is of importance for large array structures. 


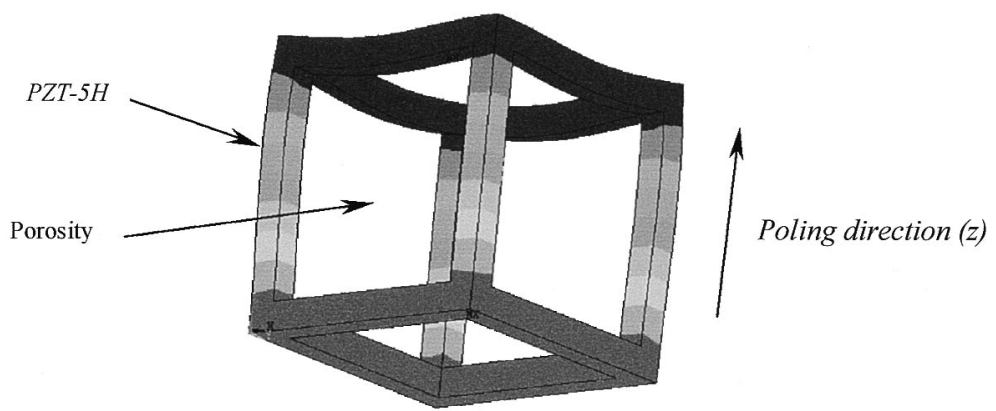

Figure 2. Diagram of the numerical cell used in finite element modelling. Porosity was systematically varied and the pore volume was filled with solid structural elements to simulate impregnation of the piezoelectric with a polymer. In this figure, the graded colours indicate the potential difference through the material under an applied hydrostatic stress.

\section{Processing Costs}

Processing costs for open porosity piezocomposites will be considerably lower than for high density materials and 1-3 manufacturing costs. Fabrication of porous 3-3 structures by use of polymer reticulated foam precursors, spray drying, or via sintering of highly calcined agglomerates will be relatively low. Similarly, since near net shaping will be possible machining costs are likely to be a minor consideration, leading to cost effective device fabrication.

In summary, the advantages of 3-3 open porosity piezocomposites are high hydrostatic figures of merit $\left(\mathrm{d}_{\mathrm{h}}\right.$ and $\left.\mathrm{g}_{\mathrm{h}}\right)$, low density, low acoustic impedance and low production costs. Compared to 0-3 and 1-3 piezocomposites, considerably less work has been undertaken on 3-3 materials. There have been some investigations of porous materials produced via various techniques and the resulting dependence of hydrostatic figures of merit on porosity volume fraction $[4,11]$. While porosity in 3-3 is often in the form of air, the material is susceptible to depth sensitivity at high volume fractions of porosity, possibly due to the poor mechanical properties of highly porous ceramics. The porous ceramics are also brittle in nature with low resistance to mechanical shock. To reduce these difficulties the impregnation of the pore space with a flexible polymer has been considered [8].

The aim of this work is to use finite element modelling to investigate the important microstructural and materials parameters in determining the performance of 3-3 piezocomposites, particularly in terms of the figures of merit $d_{h}$ and $g_{h}$. While modelling has been used to optimise 1-3 piezocomposites [13], work to date has made little attempt to tailor the performance of the 3-3 materials by design of suitable microstructures or use of the most appropriate materials. The design parameters include, porosity volume fraction, pore morphology, choice of polymer material (elastic modulus and Poisson's ratio) and choice of piezoelectric material $\left(\mathrm{d}_{33}, \mathrm{~d}_{31}\right.$ and $\left.\epsilon_{33}\right)$.

\section{Numerical Modelling}

A co-continuous microstructure of piezoceramic and porosity was developed using ANSYS 5.4 [17], a commercial finite element package. The numerical cell was based on the work of Breslin et al. [18], which has been used to model the mechanical behaviour of co-continuous structural ceramics. A typical numerical cell is shown in Figure 2. Linear piezoelectric behaviour was modelled as a static analysis using specific coupled field elements that take into account the coupled interaction of structural deformation and electrical fields in these particular materials [17]. For piezoceramics to be used in practice it is 'poled', a process which aligns the domains within the material. The material parameters 


$$
\begin{aligned}
& {[c]=\left[\begin{array}{cccccc}
12.6 & 8.0 & 8.4 & 0 & 0 & 0 \\
& 12.6 & 8.4 & 0 & 0 & 0 \\
& & 11.7 & 0 & 0 & 0 \\
& & & 2.3 & 0 & 0 \\
& & & & 2.3 & 0 \\
& & & & & 2.35
\end{array}\right] \times 10^{10} \mathrm{~N} \mathrm{~m}^{-2}} \\
& {[d]=\left[\begin{array}{ccc}
0 & 0 & -274 \\
0 & 0 & -274 \\
0 & 0 & 593 \\
0 & 0 & 0 \\
0 & 741 & 0 \\
741 & 0 & 0
\end{array}\right] x 10^{-12} m V^{-1} \quad \frac{[\varepsilon]^{s}}{\left[\varepsilon_{0}\right]}=\left[\begin{array}{ccc}
1700 & 0 & 0 \\
0 & 1700 & 0 \\
0 & 0 & 1470
\end{array}\right]}
\end{aligned}
$$

TABLE 1. Piezoelectric material parameters of PZT-5H [19], a commercially available piezoceramic. The matrix [c] denotes the stiffness matrix, $[\mathrm{d}]$ is the piezoelectric strain matrix and $[\varepsilon]^{s}$ is the permittivity at constant strain matrix, where $\varepsilon_{0}=8.85 \times$ $10^{-12} \mathrm{~F} \mathrm{~m}^{-1}$. The density of the piezoelectric material was $7500 \mathrm{~kg} \mathrm{~m}^{-3}$. The poling direction is in the 3 (or $\mathrm{z}$ ) direction for the materials data used in this format.

are therefore anisotropic and are in the form of matrices. Piezoelectric data [19] for PZT-5H were used in the model, as it is a commercially available soft lead zirconate titante ceramic that is widely used for sensor applications. The stiffness, permittivity and piezoelectric strain matrices used in this modelling are shown in Table 1.

Changing the pore size of the numerical cell, shown in Figure 2, while keeping the dimensions of the cell constant allowed the porosity volume fraction to be systematically varied. The hydrostatic figure of merit $\mathrm{g}_{\mathrm{h}}$, which describes the performance as a passive hydrophone was calculated from the electric field generated within the model per unit hydrostatic stress under open circuit conditions [13]. The figure of merit $\mathrm{d}_{\mathrm{h}}$, which describes the materials as an active device, was calculated from the net hydrostatic strain generated per unit electric field. To simulate the impregnation of the porosity with a polymeric material, the pore void was replaced with structural solid elements and the figures of merit recalculated. The effect of elastic modulus and polymer Poisson's ratio on the hydrophone figures of merit was examined by altering the mechanical properties of the polymer. For the elastic modulus, a 2-10GPa range was examined which are typical values of hard and soft set epoxies and polyurethane, assuming it is a purely isotropic material. The Poisson's ratio of the polymer was varied from 0.5 (zero volume change) to negative values as there has been interest in incorporating negative Poisson's ratio materials in piezocomposites [20]. Finally, to simulate whether the pore morphology has an effect of the performance of the 3-3 piezocomposite, the pore was elongated perpendicular and parallel to the poling direction of the piezoceramic to a variety of aspect ratios.

\section{$\underline{\text { Numerical Results and Discussion }}$}

Figure 3 shows the model results of the effect of pore volume fraction on the hydrostatic figures of merit $d_{h}$ and $g_{h}$. Both $d_{h}$ and $g_{h}$ for porous 3-3 piezocomposite are greater than that of the dense piezoceramic 


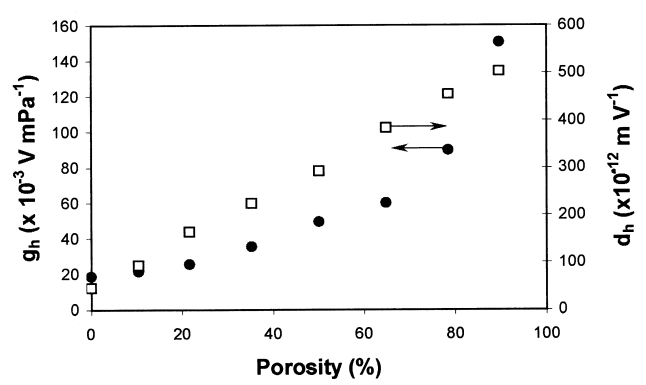

Figure 3. Graph of $d_{h}$ and $g_{h}$ versus porosity volume fraction (no polymer phase). Significant increases in $d_{h}$ and $g_{h}$ are observed compared to the dense ( $0 \%$ porosity) piezoceramic.

material, indicated by the $0 \%$ porosity area of the graph. It is seen that $\mathrm{d}_{\mathrm{h}}$ increases linearly with porosity volume fraction. As the pore volume fraction approaches $100 \%$ the value of $d_{h}$ approaches the $\mathrm{d}_{33}$ value (strain per unit field in the direction of poling) which is $593 \times 10^{-12} \mathrm{~m} \mathrm{~V}^{-1}$ for PZT-5H. This is due to the effective $d_{31}$ of the material (strain normal to the poling direction) decreasing at a considerably higher rate with porosity volume fraction compared to $d_{33}$. This indicates that materials with high $\mathrm{d}_{33}$ coefficients are candidate materials for use in 3-3 piezocomposites. The model predicts large increases for the hydrophone figure of merit $g_{h}\left(=d_{h} / \epsilon^{T}{ }_{33}\right)$ which is due to the increase in $d_{h}$ and the decrease in effective permittivity of the material as porosity replaces the high permittivity ceramic. These results agree well with the experimental observations of Guillaussier et al. [11] in terms of the typical values for the figures of merit observed, that $d_{h}$ approaches the $d_{33}$ value at high porosity volume fractions and the form of the $d_{h}$ and $g_{h}$ curves as a function of porosity volume fraction. This demonstrates that the hydrostatic figures of merit $d_{h}, g_{h}$ and $d_{h} \cdot g_{h}$ can be considerably increased by incorporation of porosity into a 3-3 structure.

The model does not take into account any possible damage of the porous materials as a function of water depth and associated pressure. It has been found that for porous ceramic-air systems, the figures of merit are depth sensitive at porosity volume fractions greater than 50\% [11]. To reduce the depth sensitivity of highly porous ceramics and provide some flexibility to the composite, the materials can be impregnated with a polymer to reduce damage under high hydrostatic stress. Figure 4 is a graph of $d_{h}$ versus polymer Poisson's ratio for an impregnated piezocomposite with $50 \%$ porosity with a variety of elastic moduli (the $\mathrm{g}_{\mathrm{h}}$ data have been removed for the sake of clarity). It can be seen from Figure 4 that $d_{h}$, and therefore $g_{h}$, is maximised by using a polymer of low elastic modulus and low Poisson's ratio. The elastic modulus trend observed is due to the increasingly stiff polymer reducing the stress

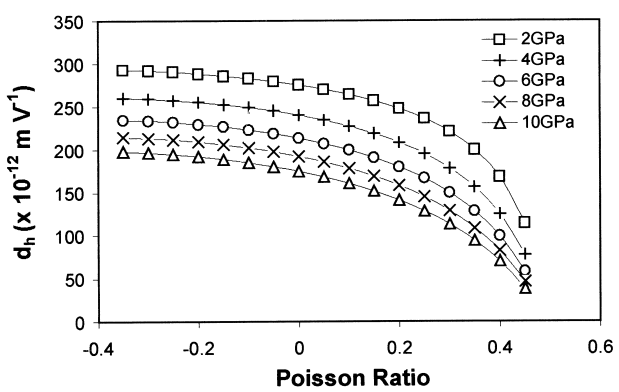

Figure 4. Graph of $d_{h}$ versus polymer Poisson's ratio for polymers of different elastic modulus. The legend indicates the value of elastic modulus. $d_{h}$ (and therefore $g_{h}$ ) increase with decreasing Poisson's ratio and decreasing elastic modulus. 


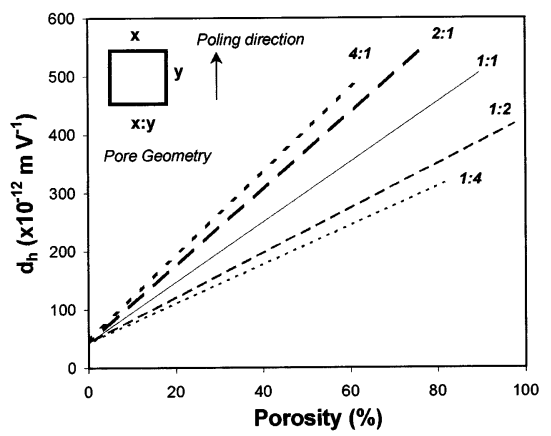

Figure 5. Graph of $d_{h}$ versus porosity for pores of different aspect ratio. $d_{h}$ is increased by incorporating pores elongated perpendicular to the poling direction.

transfer into the piezoceramic material in the passive mode and hindering the development of strain of the material in the active mode. As the Poisson's ratio of the polymer phase is reduced the lateral contraction is reduced, enhancing the 'piston-like' motion of the composite and increasing $\mathrm{d}_{\mathrm{h}}$. This has been examined in 1-3 piezocomposite research where there is interest in using negative Poisson's ratio materials as the polymer filler [13,20-22]. For 3-3 piezocomposites, no benefits are observed in terms of $d_{h}$ and $g_{h}$ in using these new materials, as seen in Figure 3, where $d_{h}$ is only sensitive to Poisson's ratio values in the region of 0.5 to 0.1 . This is a consequence of the three-dimensional connectivity of the ceramic, which restricts any benefit of using a negative Poisson's ratio material compared to the one-dimensional ceramic connectivity of the 1-3 piezocomposites.

In addition to the piezoelectric material, polymer material and pore volume fraction, the morphology of the pores within the piezocomposite has an influence of the hydrostatic figures of merit. Figure 5 is a graph of $d_{h}$ versus pore volume fraction for pores with a variety of aspect ratios. Figure 5 shows that there is an increase in $d_{h}$ as the pores become increasingly more elongated perpendicular to the direction of poling. This is due to the elongated pores reducing the lateral expansion or contraction, associated with the $\mathrm{d}_{31}$, whilst maintaining the $\mathrm{d}_{33}$ for a given porosity. Elongated pores have been produced in a ceramic by coating elongated polymer foams with a ceramic slurry and heat treating to remove the polymer and sinter the ceramic [10].

\section{Conclusions}

Based on the finite element modelling of a co-continuous 3-3 piezocomposite the following conclusions can be drawn.

1. Finite model predictions are in good agreement with published experimental data. The modelling technique described is an appropriate methodology for modelling microstructural and materials parameters determining the behaviour of 3-3 piezocomposites.

2. The hydrophone figures of merit $\left(\mathrm{d}_{\mathrm{h}}\right.$ and $\left.\mathrm{g}_{\mathrm{h}}\right)$ increase with increasing porosity volume fraction and are considerably greater than those of fully dense materials.

3. The value of $d_{h}$ approaches the $d_{33}$ value as porosity volume fraction increases. This implies that piezoelectric materials with high $\mathrm{d}_{33}$ coefficients are candidate materials for 3-3 piezocomposites.

4. If the ceramic matrix is impregnated with a polymer material, the hydrostatic figures of merit increase with decreasing polymer stiffness. 
5. Reducing the Poisson's ratio of the polymer phase increases the hydrostatic figures of merit. At low values of Poisson's ratio $(<0.1)$ no significant benefits were observed. The results obtained indicate that the incorporation of a negative Poisson polymer into a 3-3 piezocomposites would not significantly improve performance $\left(\mathrm{d}_{\mathrm{h}}\right.$ and $\left.\mathrm{g}_{\mathrm{h}}\right)$.

6. In addition to pore volume fraction, pore morphology has an effect on the behaviour of the composite material. Pores of high aspect ratio aligned perpendicular to the poling direction have been shown to enhance the hydrostatic sensitivity of these materials.

\section{Acknowledgments}

The authors would like to acknowledge EPSRC and DERA for funding this project.

\section{$\underline{\text { References }}$}

1. G. H. Haertling, J. Am. Ceram. Soc. 82, 797 (1999).

2. T. F. Murray, Dungan RH. Ceram. Ind. 82, 74 (1964).

3. A. I. Kingon and J. B. Clark, J. Am. Ceram. Soc. 66, 253 (1983)

4. G. Galassi, E. Roncar, F. Craciun, A. Bettucci, F. Farrelly, and A. Alippi, Fourth Euro Ceramics, Electroceramics. 5, 25 (1995).

5. K. Mizumura, Y. Kurihara, H. Ohashi, S. Kummamoto, and K. Okuno K, Jpn. J. App. Phys. 30, 2271 (1991).

6. K. Ina, T. Mano, S. Omura, and K. Nagata, Jpn. J. App. Phys. 33, 5381 (1994).

7. T. Hayashi, S. Sugihara, and J. Okazaki, Jpn. J. App. Phys. 30, 2243 (1991).

8. D. P. Skinner and R. E. Newnham, Cross LE. Mat. Res. Bull. 13, 599 (1978).

9. R. E. Newnham, D. P. Skinner, and L. E. Cross, Mat. Res. Bull. 13, 525 (1978).

10. V. F. Janas and A. Safari, J. Am. Ceram. Soc. 11, 2945 (1995).

11. P. Guillaussier and C. A. Boucher, Ferroelectrics, 187, 121 (1996).

12. H. R. Gallantreev, Brit. Ceram. Proc. 41, 161 (1989).

13. J. Bennett and G. Hayward, IEEE Trans. Ultra. Ferro. Freq. Cont. 44, 565 (1997).

14. L. V. Gibansky and S. Torquato, Structural Optimisation. 13, 23 (1997).

15. O. Sigmund, S. Torquato, and L. A. Iaksay, J. Mat. Res. 13, 1038 (1998).

16. F. Constantinescu, P. Nicolau, E. Dimitriu, Vasiliu, C. Bunescu, F. Craciun, A. Bettucci, F. Farrelly, and A. Allippi, Fourth Euro. Ceramics. 5, 145 (1995).

17. ANSYS, Inc., ANSYS Theoretical Manual, Version 5.4, Houston, PA, 1998.

18. M. C. Breslin, B. Starck, L. Xu, G. S. Daehn, and H. L. Fraser, Fourth Euro. Ceramics. 3, 71 (1996).

19. H. A. Kunkel, S. Locke, and B. Pikeroen, IEEE Trans. Ultra. Ferro. Freq. Cont. 37, 316 (1990).

20. W. A. Smith, Ultrasonics Symposium. 1, 661 (1991).

21. M. Avellaneda and P. J. Swart, J. Acous. Soc. Am. 103, 1449 (1998).

22. L. V. Gibiansky and S. Torquato, J. Mech. Phys. Sol. 45, 689 (1997). 